\title{
Essais
}

ESSAIS

Revue interdisciplinaire d'Humanités

$11 \mid 2017$

Fictions de l'identité

\section{Réécrire Antigone : le recours au mythe en temps de trouble}

Fanny Blin

\section{(2) OpenEdition}

1 Journals

Édition électronique

URL : http://journals.openedition.org/essais/3351

DOI : 10.4000/essais.3351

ISSN : 2276-0970

Éditeur

École doctorale Montaigne Humanités

Édition imprimée

Date de publication : 15 mai 2017

Pagination : 53-68

ISBN : 979-10-97024-01-7

ISSN : 2417-4211

Référence électronique

Fanny Blin, «Réécrire Antigone : le recours au mythe en temps de trouble », Essais [En ligne], 11 | 2017, mis en ligne le 14 octobre 2020, consulté le 21 octobre 2020. URL : http://journals.openedition.org/ essais/3351; DOI : https://doi.org/10.4000/essais.3351 


\section{Réécrire Antigone : le recours au mythe en temps de trouble}

\section{Fanny Blin}

Les œuvres littéraires traitant de la Guerre d'Espagne, épisode critique dont l'écho se prolonge au moins jusqu'à la Transition démocratique, ont particulièrement mis en question l'idée d'unité nationale. Les discours se sont peu à peu codifiés autour d'un ensemble d'images récurrentes pour représenter cet épisode clivant de l'histoire. Naturellement, les mythes et les grandes tragédies alimentent ces topö̈, à l'instar d'Antigone, qui hante la dramaturgie espagnole depuis 1939 jusqu'aux années 1980. Avec près de vingt "réécritures " de la tragédie de Sophocle, une telle omniprésence d'Antigone dans les productions théâtrales d'après-guerre n'a rien d'anodin ${ }^{1}$. En effet, la période correspond à d'éminents troubles politiques dans le pays, après le conflit armé qui a divisé la nation et débouché sur une longue dictature. Un tel contexte a nécessairement remodelé les modes d'expression littéraires, et le cas de la reprise des tragédies grecques antiques est particulièrement intéressant pour étudier comment le théâtre - en tant que texte et en tant que spectacle vivant - participe à des processus de questionnements identitaires. La profonde division au sein de la société, provoquée par la guerre civile, a forgé un clivage profond. Selon Beatriz Lomas Lozano, "L'identité nationale de l'Espagne d'après-guerre se cimente à partir de l'héroïsme de guerre du passé et la construction d'un imaginaire de la part des deux camps ${ }^{2}$. "Dans cette mesure, la manière dont le théâtre espagnol s'est saisi du sujet politique qu'est Antigone pour questionner une identité collective mise à mal s'intègre pertinemment à cette réflexion commune sur la participation de la fiction aux reconstructions identitaires (individuelles et nationales).

1 Voir Luciano Curreri, «La guerre civile espagnole entre fiction et réalité », in Interférences littéraires, Nouvelle série, $\mathrm{n}^{\circ}$ 3, "Les écrivains et le discours de la guerre ", François-Xavier Lavenne et Oliver Odaert (éd.), novembre 2009, p. 217-225.

2 «La identidad nacional de la España de la posguerra se cimienta a partir de la heroicidad bélica del pasado y la construcción de un imaginario por parte de ambos bandos. ", Beatriz Lomas Lozano, Reflexiones sobre el poder en Luis Riaza, Francisco Nieva y José Luis Alonso de Santos, Chapel Hill, ProQuest, 2007, p. 15. 
La littérature espagnole actuelle est fortement nourrie par un retour constant sur ce contexte historique troublé et la mémoire de la guerre civile, toujours problématique. Si cette parole et ce débat sont davantage possibles aujourd'hui, les modalités d'expression tissaient un système d'entraves profondes pendant la dictature. Après les clivages créés par la guerre dans le corps de la nation, le franquisme a reposé sur un rapport de domination entre vainqueurs et vaincus. Cette dualité était récurrente dans les discours politiques d'après-guerre, qui ont construit la fiction des deux camps imperméables, binaires, dont la victoire ou la défaite étaient supposément constitutives d'une identité collective. Toutefois il faut souligner qu'un seul "camp " était audible dans les années 1940 et 1950. En effet, la censure imposée a rendu délicate, voire impossible, la représentation de cette guerre par les vaincus. La structure de cette situation politique n'a pas manqué de faire penser à celle de la tragédie des Labdacides. L'image des frères Étéocle et Polynice s'entretuant pour le trône de Thèbes est parallèle au conflit fratricide entre républicains et nationalistes. Mais les échos ne s'arrêtent pas là : les problèmes abordés dans la tragédie sophocléenne, tels que la sépulture, l'exil et la tyrannie, en font le paradigme idoine pour exprimer le trouble contemporain. Par conséquent, comparer la période d'après-guerre à ce récit mythique est un lieu commun. Ce détour par le mythe a justement permis à certains créateurs espagnols de re-présenter leur conflit contemporain. L’omniprésence de la figure d'Antigone, érigée en symbole de la résistance, est particulièrement frappante dans la dramaturgie espagnole. Son histoire est mise en scène par plus de vingt dramaturges espagnols entre 1939 et la décennie 1980. Cela confirme le lien qui existe entre le conflit civil - puis la dictature - et cette tragédie inspirée du mythe. Précisément, ce procédé littéraire de réécriture (ou récriture), qui a été un véritable phénomène, a participé aux reconstructions identitaires, cruciales en cette période trouble.

Au-delà des coïncidences entre mythe et histoire, il semble intéressant d'observer comment la figure Antigone a cristallisé l'expression de la crise sociétale, identitaire et politique du $\mathrm{XX}^{\mathrm{e}}$ siècle en Espagne. Non seulement les adaptations de la tragédie de Thèbes par les dramaturges espagnols reflètent le conflit contemporain, mais elles sont le produit de ce trouble, qui influence les modalités d'expression. Parfois pour glorifier les vaincus, parfois pour caricaturer le pouvoir, mais aussi afin d'éviter la censure, les auteurs ont développé des stratégies littéraires variées. Les symboliques mises en œuvre interrogent l'identité collective et individuelle à travers le processus éminemment théâtral de l'identification. Il s'agit de reconstruire ce qui a été pulvérisé par le drame de la guerre civile : une image de $\operatorname{soi}^{3}$, tout d'abord. Cette " reconquête " s'incarne dans la crise identitaire traversée par l'héroïne, et révèle également

3 Dans la perspective développée par Martina Avanza et Gilles Laferté, dans "Dépasser la "construction des identités" ? Identification, image sociale, appartenance ", Genèses, n 61, 2005, p. 134-152. 
les tentatives de reconstructions identitaires (à l'échelle des groupes) de la part des auteurs. En outre, la dimension cathartique de la parole libérée, permise par l'entremise d'une figure qui incarne cette liberté et qui est étroitement liée à la psychanalyse, débouche sur l'affirmation du sujet individuel.

Quelques jalons sont essentiels afin d'entrevoir le panorama global des réécritures de l'Antigone de Sophocle : entre 1935 et 1989, une vingtaine de pièces s'inspirent de la tragédie. Elles ont été écrites en trois langues (castillan, catalan et galicien), ce qui suppose par définition des espaces de réception différents. La plupart de ces pièces n’ont été éditées et représentées pour la première fois qu'après la mort de Franco, souvent dépeint sous les traits du tyran Créon. Cette réflexion se concentre sur six œuvres en particulier : Antígona, du catalan Salvador Espriu, écrite en 1939, Antígona, de José María Pemán, représentée sous Franco en 1945; La Sangre de Antígona, de José Bergamín, écrite en exil dans les années 1950 et publiée en 1983 ; La Tumba de Antígona, de María Zambrano, rédigée en exil également ; Antígona entre muros, de José Martín Elizondo, pièce de l'exil éditée pendant la Transition démocratique, et enfin Antígona... jcerda!, de Luis Riaza, écrite pendant la dictature et publiée seulement après. Le regard porté par ces dramaturges sur le parcours d'Antigone présente une riche variété. Il faut souligner que la réécriture de cette tragédie n'est pas l'apanage des opposants au Régime. Ainsi, on trouve des Antigones "chrétiennes ", des œuvres " de l'intérieur ", par opposition à l'exil, des Antigones révolutionnaires et des porte-drapeaux du nationalisme catalan. Les partis pris esthétiques et idéologiques sont très divers et témoignent précisément de la complexité d'une époque. Par conséquent, l'appropriation de la figure mythique constitue un enjeu identitaire et son traitement varie autant que les formes d'écriture.

\section{Représenter le conflit : la scène comme espace du possible}

\section{Une tragédie plus que jamais contemporaine}

Dans un contexte où la guerre civile oblige les artistes espagnols à explorer de nouvelles voies d'expression du trouble et à repenser leur identité individuelle et collective, la tragédie, fondée sur la catharsis, est un vecteur de messages particulièrement propice. La situation politique de la guerre civile - puis de la dictature - plonge le théâtre dans une crise qui pousse les dramaturges à trouver des cadres de compréhension du monde, d'où le recours au mythe. Touchant à l'inénarrable, la violence de la Guerre civile affecte nécessairement l'identité individuelle et collective, et requiert la réinvention de modalités pour l'exprimer. Une telle quête identitaire a débouché sur des réécritures de textes anciens, de paraboles à dimension universelle, comme les tragédies grecques. Indéniablement, il y a eu un véritable phénomène de résurgence de nombreux mythes dans les écrits de cette époque, comme le souligne la 
critique Maria Josep Ragué i Arias 4 . Parmi ceux-là, les destins d'CEdipe et de sa fille Antigone ont été les plus exploités en Espagne, particulièrement au théâtre. Plusieurs facteurs expliquent cette focalisation sur ces figures précises : naturellement, il faut considérer l'influence française d'Anouilh, qui avait fait d'Antigone un symbole de la résistance pendant la Seconde Guerre mondiale. De surcroît, cette tragédie aborde la question de la sépulture des morts après une lutte fratricide, un thème qui parle aux Espagnols plus que jamais. Le conflit armé a fait de nombreux morts et ceux du camp républicain n'ont pas eu droit à une sépulture digne : la situation de la tragédie antique se reproduit en Espagne, et réactive donc ce mythe. Selon Rose Duroux, si « les fosses communes hantent l'imaginaire collectif espagnol : rien d'étonnant à ce que le cri d'Antigone s'y répercute dans la littérature comme au Parlement ${ }^{5}$ ». Par exemple, José Bergamín souligne dans sa réécriture d'Antigone le caractère contre-nature d'une sépulture différente pour les deux frères, en insistant sur cette idée du retour à la terre. Il écrit notamment : «Leur sang est réuni dans la terre mais leurs corps gisent séparément pour toujours, par la volonté des vivants $^{6} »$. Il est d'ailleurs intéressant de relever que Bergamín met en scène le moment où Antigone répand la terre sur le cadavre de son frère - fait qui n'est qu'indirectement relaté dans la tragédie sophocléenne. Manifestement, ce choix esthétique correspond à un acte militant, tendant à construire une réalité alternative. En décidant de créer cet épisode, Bergamín donne à voir le geste de la sépulture, hautement symbolique en cette période. La fiction théâtrale fonctionne là comme un processus thérapeutique puisque l'acte proscrit est réalisé sur scène, ostensiblement. Les réécritures d'Antigone participent donc à ce " tombeau littéraire ", construit pour les morts dont la mémoire n'a pas pu être célébrée. En cela, elle est cathartique, puisque l'identification est fondamentale dans ces œuvres engagées.

\section{Se définir individuellement à travers l'identification à un camp}

Ces résurgences émanent de la nécessité de se redéfinir en tant qu'individu, nation ou camp, après la scission opérée par la guerre. En cette période noire de l'histoire espagnole, on observe une profusion de productions artistiques autour du concept d'identité. Au théâtre en particulier, les œuvres qui se centrent sur la mise en scène de conflits identitaires, à échelle individuelle

4 María Josep Ragué i Arias, Lo que fue Troya: los mitos griegos en el teatro español actual, Madrid, Asociación de autores de teatro, 1992.

5 Rose Duroux et Stéphanie Urdician, "Antigone. Retours sur une fascination. ", Les Antigones contemporaines (de 1945 à nos jours), Clermont Ferrand, Presses Universitaires Blaise Pascal, 2010, p. 21.

6 José Bergamín, La Sangre de Antígona, misterio en tres actos, in María Teresa Santa María Fernández, El teatro en el exilio de José Bergamín, Barcelone, Presses universitaires de l'Universitat Autònoma de Barcelona, 2001, p. 383-426. 
ou collective, rencontrent le plus de succès. Les thématiques les plus reprises sont celles de la marginalité, de la violence, et de la solitude. Cette réflexion sur l'identité individuelle s'inscrit dans le sillon antique, par la reprise de tragédies que les dramaturges actualisent pour correspondre à leur époque. Les archétypes mythiques constituent alors une « béquille " pour la représentation des grands conflits traversés par les Espagnols. Une crise identitaire a en effet affecté la nation, comme le sujet individuel, après cette guerre qui n’a défini les hommes qu'en fonction de leur appartenance à un clan, à un camp politique, et a déterminé leur sort, leur destin : celui d'exilé, celui de condamné à mort, celui de soutien du régime... Le chaos a poussé chacun à se définir en fonction de ces critères qui peu à peu ne sont plus valides, car trop simplistes. Cependant ils continuent d'être en vigueur jusqu'aux années soixante-dix, revenant sur le devant de la scène à l'occasion des débats sur la mémoire de la guerre civile pendant la Transition démocratique. L'imbrication entre l'histoire espagnole du XX siècle et l'histoire mythifiée de la Grèce antique sera alors sans cesse soulignée. Maintes fois, les artistes et les politiques compareront la Transition à la Guerre de Troie ou à d'autres épisodes mythiques. La rébellion d'Antigone est donc reprise, réécrite, pour réfléchir sur l'identité individuelle (avec la représentation d'un conflit au sein du personnage principal, entre son obligation fraternelle et son obligation civile) mais aussi sur l'identité collective (en tant que nation désunie à reconstruire). La mise en scène du conflit entre le devoir religieux - ou l'amour filial, selon les pièces et le devoir d'obéissance aux lois civiles permettent la projection. La dimension visuelle du théâtre permet de projeter sur scène le trouble qui se joue dans la psyché des Espagnols et de pallier ainsi la difficulté à l'exprimer. Cette représentation scénique confronte enfin les discours des deux camps opposés, via l'incarnation de ceux-ci dans des personnages archétypiques. Ainsi, Créon représente l'autorité impitoyable et Antigone se fait la porte-parole de ceux qui se perçoivent comme opprimés. Le caractère binaire de ce schéma favorise la compréhension de dynamiques historiques qui posent des problèmes de représentation, en les synthétisant.

\section{La rhétorique d'opposition mise en scène : de "l'Autre » à l'ennemi}

Écrire des Antigones engagées répond au besoin de se définir par rapport à l'ennemi, mais le masque du mythe antique permet aussi d'éviter la censure. Il faut souligner que si les dramaturges passent par le filtre du mythe, c'est aussi parce que cette distance prise par rapport à la réalité contemporaine permettait de critiquer leur époque en contournant la censure d'après-guerre. Ainsi, il était envisageable de diffuser un message engagé à demi caché, et légitimé par le caractère fondamental de ces éléments mythiques dans la culture occidentale. Les œuvres qui nous intéressent dépeignent Antigone comme le symbole de la résistance civile, mais aussi de la réconciliation post- 
lutte fratricide. En particulier, il est marquant de constater à quel point cette figure se recompose à mesure que passent les années et que les problématiques évoluent. En effet, plus on s'éloigne de la guerre civile, plus cette figure féminine porte des messages d'apaisement, et non plus de résistance. Elle s'achemine peu à peu, pour la majeure partie des pièces, vers des discours de pardon et de démocratie. Paradoxalement, les Antigones de la Transition démocratique semblent plus radicales, plus subversives. Cela s'explique parfois par la déception suscitée par la Transition, ou par le décalage entre l'écriture et l'édition. L'ouverture relative de la fin du franquisme, avec la nouvelle Ley de Prensa, a en effet été marquée par la publication d'œuvres contestataires qui avaient été écrites dans les années précédentes, comme l'Antigona...cerda de Luis Riaza, pièce très provocatrice. Ce phénomène s'accentuera encore après 1977. Si Antigone jouit d'un tel engouement, c'est parce que la métaphore constituait un biais commode pour ceux qui écrivaient pendant la dictature. Par conséquent, les personnages des mythes séculaires ont porté les masques de leurs revendications. Ainsi l'Antigone de José Martín Elizondo constitue ce qu'on peut appeler une parabole de la dictature : Antígona entre muros, écrite pendant son exil en France, dénonce les dérives dictatoriales. L'action se déroule en Grèce dans une prison pour femmes, où les détenues répètent une pièce intitulée Antígona, qui est " une œuvre interdite " comme le précisent les captives. Le personnage principal est construit comme une victime de la lutte clandestine contre le Régime. L'écriture est grandement symbolique : l'oppression politique y est par exemple représentée par les chiens des gardes de la prison. La distance prise avec la tragédie de Sophocle montre que la figure mythique est un outil. Quelques années plus tard, la nécessité d'enterrer la hache de guerre devenait un enjeu majeur. Il s'agissait de construire l'image, l'identité d'une Espagne unie et démocratique. Antigone a été reconfigurée à cette époque, tantôt comme symbole de réconciliation d'une famille déchirée et meurtrie, tantôt mise en scène dans ses travers, pour dénoncer le revirement idéologique qui a eu lieu lors de la Transition, de la part des vaincus. Riaza fait ainsi d'Antigone une fausse rebelle, qui a vendu son âme sur l'autel du conformisme. Cette pièce participe à la redéfinition identitaire, et au positionnement individuel (qui s'écrit à l'échelle du personnage et à celle de l'écrivain). Reprendre une tragédie antique pour décrire sa tragédie nationale n’est donc pas un paradoxe : passer par un mythe issu d'un espace-temps lointain pour dépeindre son époque est en réalité un moyen d'escamoter la critique frontale qui serait immédiatement censurée. En outre, il s'agit d'un appui pour la pensée : la prise de distance que cela suppose est bénéfique pour penser la crise contemporaine à chaud. Le détour par le mythe permet de formuler, mais aussi de représenter spatialement le conflit, sur scène. Ainsi, la projection de l'opposition s'opère et celle-ci peut s'incarner dans des personnages qui synthétisent le discours (en le simplifiant et le rendant accessible) afin de pouvoir dire le trouble, et surtout le représenter. 


\section{La mémoire des siens}

\section{Une parole dissonante contre le pacte du silence et la mémoire officielle}

La richesse du personnage mythique d'Antigone se prête judicieusement à l'expression de la crise, en tant que figure de la mémoire. Source d'inspiration privilégiée des Espagnols, Antigone est l'ensevelisseuse qui ne recule devant rien pour honorer la dépouille de son frère. Comment cette femme fictive, enracinée dans un contexte antique, a pu porter si idéalement une cause contemporaine ? Cela est dû précisément à la tension entre l'histoire de la dictature espagnole et la crise identitaire qu'elle a engendrée et alimentée sur le plan de l'image que les citoyens se faisaient de leur nation. Le pacte de silence établi lors de la Transition démocratique interdit le retour sur les épisodes traumatiques qui ont divisé les Espagnols, créant alors une parole impossible, encore une fois censurée, après des années de contrôle des productions historiographies et frictionnelles. L'impossible analyse du passé troublé de la Guerre civile compromet l'unité contemporaine dans les années 1970 et 1980 . Or il y a une profusion de réécritures à cette époque, ce qui montre bien que cette tragédie constitue le cadre privilégié de l'expression de cette crise qui touche à l'identité nationale. Si l'on en croit le psychanalyste Vincent Estellon, "[l]'identité se construit dans le lien, elle "tient" grâce à la mémoire. Et Antigone n’oublie pas le passé7 ». Ce détour par une tragédie antique qui met en scène une femme qui s'oppose à la mémoire officielle du conflit est idéal pour projeter un sens sur une époque contemporaine perturbée. Le discours officiel décidé unilatéralement par le cœur du pouvoir y est fortement dénoncé, à travers des œuvres d'autant plus subversives à partir de 1975. La politique mémorielle du Régime y est caricaturée sous les traits du tyran Créon, qui choisit lequel des deux frères mérite les honneurs et le deuil de la communauté. Les adaptations d'Antigone les plus récentes se caractérisent donc par un discours plus subversif et plus libre afin de dénoncer la manière dont le Régime dictatorial a imposé un discours mémoriel tronqué et verrouillé. L'écriture elle-même consiste alors en un acte de rébellion similaire à celui de l'héroïne, surtout lorsqu'il s'agit d'écrire dans une langue qui avait été proscrite par le pouvoir pendant les premiers temps de la dictature.

Ce personnage tragique, en tant qu'incarnation de l'opposition, a donc permis aux dramaturges espagnols de vivifier un art censuré et de lire leur époque au prisme du mythe. Il s'agit en effet d'un symbole maintes fois exploité politiquement dans le contexte européen, et lié à l'engagement de l'écrivain, comme l’a montré notamment G. Steiner ${ }^{8}$. À ce titre, la scène centrale de la tragédie

7 Vincent Estellon, « Mémoire, genre, identité et lien social : le cri d'Antigone », Champ psychosomatique, $\mathrm{n}^{\circ}$ 58, 2010, p. 153.

8 George Steiner, Les Antigones, Paris, Gallimard, 1986. 
est souvent celle de la confrontation entre la jeune femme et Créon, qui tente de la dissuader de résister. Dans la pièce de Elizondo, la résistance ne porte plus sur l'ensevelissement d'un corps, mais l'accent est mis sur le dilemme moral des prisonnières, qui doivent ou non dénoncer leurs camarades de lutte : les véritables héroïnes sont celles qui se refusent à collaborer avec le régime tyrannique. Dans le contexte des luttes sociales, ouvrières et étudiantes qui avaient lieu en Espagne, cette œuvre fusionne classicisme et modernité pour justifier et défendre la cause des opposants au pouvoir. En effet, la comparaison des revendications contemporaines avec la lutte d'Antigone attribue un caractère universel et légitime à ces manifestations. Ce que dénonce cette pièce, écrite en exil en 1969, c'est à la fois la répression de ces tentatives de dissidence actuelles, mais également l'écrasement visant à l'annihilation des perdants de la Guerre civile de la part du gouvernement franquiste depuis les années 1940. Malgré l'évolution de son discours officiel et son semblant d'ouverture, Elizondo souligne la constance de la répression et de l'autoritarisme qui fait taire les voies dissidentes à l'instar de Créon qui emmure Antigone. Cette œuvre, publiée en France, depuis un terrain plus favorable, peut compter parmi ces «Antigones de l'exil » qui ont marqué les réécritures contemporaines.

\section{L'expérience de l'exil ou l'identité mise à mal}

Dans la tragédie grecque, Antigone a parcouru un long chemin initiatique avant de s'opposer frontalement à son oncle : ce chemin passait par l'accompagnement de son père CEdipe dans son exil. C'est pourquoi les écrivains espagnols engagés qui ont été bannis par le pouvoir dictatorial s'identifient à elle. À travers leurs essais sur cette condition de vie, on peut mesurer l'ampleur de la crise identitaire provoquée par un tel déracinement des exilés. Ce qui est marquant à ce propos, c'est le rapport à la terre (terre d'origine) qu'implique ce bannissement. L'exil suppose en effet une tension particulière vis-à-vis de la terre, dans la mesure où un individu se sent enraciné et lié à son lieu de naissance. Pour les exilés, cette impossibilité d'y retourner entraîne un déchirement fondé sur la négation de leur autochtonie, qui est une part constitutive de leur identité. Une telle tension envers la terre d'origine s'illustre également dans l'interdiction d'y être enterré, prononcée elle aussi par le tyran : dans ces deux cas, il est impossible de retourner à sa terre propre. Or Antigone représente les deux facettes de ce rapport à la terre : exil et enterrement, deux notions d'autant plus proches que le terme espagnol destierro, qui signifie " exil », est étymologiquement formé sur l'idée du "détachement " par rapport à la terre. Exilée aux côtés de son père qu'elle guide sur des chemins hasardeux, hors de Thèbes, elle est bien évidemment connue pour son obstination à rendre à Polynice les honneurs funèbres. C'est pourquoi elle s'impose comme la figure idéale pour incarner cette déchirure identitaire et personnelle dans l'Espagne d'après-guerre. Les nombreux artistes exilés, après la guerre, ont 
beaucoup exploité ce parallèle dans leurs productions, afin de poser une parole sur cette épreuve qui questionne l'origine de l'être, et sa finitude. L'œuvre de Maria Zambrano est emblématique de cette réflexion car, après avoir philosophé sur le sens de cet exil existentiel, elle compose une pièce hybride. Celle-ci met en scène une Antigone errante, entre la vie et la mort, dans sa tombe, qui représente l'espace de l'entre-deux par excellence qu'est l'exil. La Tombe d'Antigone exploite le motif de l'espace de passage : Antigone y est suspendue entre la vie et la mort, condamnée par Créon pour son entêtement à défendre les " lois non écrites " qui l'obligeaient à enterrer son frère. La crise de l'identité, créée par la guerre, est ici symbolisée par la double impossibilité : être quelque part, et être caractérisé. Finalement, dans cette œuvre, la tombe apparaît comme un espace familier, un lieu apaisant, où le personnage parvient à se définir et à se trouver, par la parole.

\section{Antigone comme icône de la réconciliation}

L'exemple de la pièce du célèbre poète Salvador Espriu, écrite au cœur du conflit, en 1939, dans une Barcelone occupée par les troupes du «bando nacional " est assez parlant. À cause de la censure de la langue catalane, la pièce est restée inédite pendant les premiers temps de la dictature. Espriu déclare qu'il a écrit cette œuvre pour " tenter de surmonter l'esprit de la guerre civile, de ses oppositions et ses haines ${ }^{9}$ ". Le mythe est donc le prétexte dont le dramaturge se sert pour exprimer l'horreur de la guerre civile. Cette création pourrait passer pour une œuvre d'apaisement pour panser les blessures de ce déchirement national, une proposition de reconstruction à caractère identitaire qui se fonde sur la guerre, mais elle dépasse ce cadre en étant très critique. Antigone y prône une paix sociale possible, une via media entre les deux frères ennemis, une réconciliation post-guerre civile, au-delà du maintien du rapport vainqueurs/vaincus de la part du Régime. José Bergamín également construit une figure poétique de la paix dans son œuvre Le Sang d'Antigone, écrite en 1955 pendant son exil à Paris : le dramaturge a déclaré cette année-là que son œuvre constituait " un chant pour la réconciliation du peuple ${ }^{10}$ ». Il est intéressant de remarquer que dans l'œuvre de Bergamín, tout comme dans celle de Zambrano, Antigone dialogue avec les fantômes de ses deux frères : cela semble signifier que la parole dénoue le conflit, que l'écriture reconstruit alors l'identité, et peut réconcilier les opposants.

9 "Era una obra que trataba de superar el espiritu de la guerra civil, sus oposiciones y odios " (notre traduction), Salvador Espriu, cité par Rosana Torres dans " Antígona, de Espriu, se representará por primera vez en castellano en Mérida ", El País, Madrid, 10 juin 1986.

10 María Teresa Santa-María Fernández, El teatro en el exilio de José Bergamín, Barcelone, Presses universitaires de l'Universitat Autònoma de Barcelona, 2001, p. 383. 
Concernant la réconciliation mais dans un tout autre registre, José María Pemán a créé une Antigone chrétienne et martyre, présentant une trame tragique relativement fidèle à Sophocle. Il s'agit d'une Antigone rare à cette période, car c'est un parti pris pro-franquiste. Le discours officiel du régime y est transmis, insistant sur l'idée que les nationalistes ont pacifié le pays après la guerre civile. Dans cette pièce, contrairement aux accents politiques contemporains des autres réécritures espagnoles, le conflit tragique repose sur le dilemme entre le devoir religieux (celui de la sépulture) et les lois de la cité. Pemán fait d'Antigone une figure de martyre pré-chrétienne. Il retourne ainsi l'argument de ses opposants, en montrant que les véritables tyrans sont ceux qui vont à contre-sens des principes religieux. Franco apparaît alors comme le garant de la justice divine, ce qui légitime son pouvoir. L'œuvre verse dans la propagande puisque la désobéissance d'Antigone au tyran, au lieu de l'ériger en héroïne, ne fait que la pousser à sa perte. Dans les faits, la nuance est faible, mais le sens de l'écriture, l'éclairage historique contemporain apporté par Pemán présente les événements sous un angle plus prosaïque. Il est surtout montré que la jeune femme entraîne dans la mort son fiancé Hémon. Le dénouement apporte un message clair : la résistance est vaine, vide de sens. De surcroît, cette version met l'accent dès le premier acte sur la victoire militaire (sous-entendu, des troupes nationalistes) et non sur la lutte fratricide. En élevant l'histoire de son pays au rang de tragédie, Pemán justifie la victoire des «soulevés » et de Franco, et la présente comme émanant de la volonté divine.

\section{De la version contemporaine à la subversion du mythe : Antigone devient un instrument de revendication identitaire}

Les différentes identités revêtues par Antigone dans les réécritures révèlent la capacité de cette figure tragique à incarner des causes très diverses

La variété des représentations d'Antigone précédemment évoquées montre que cette figure peut être chaque fois reconstruite pour incarner un positionnement différent : le mythe a donc été adapté, au sens fort du terme. Cela met en évidence sa capacité originelle à exprimer des conflits humains et des problématiques identitaires profondes, car universelles. À partir de là, les différents traitements de la source classique par ces dramaturges traduisent l'évolution des regards sur le conflit tragique au cours de la période, depuis l'immédiat aprèsguerre jusqu'au retour de la démocratie. Ces réécritures nous livrent donc, d'une certaine manière, la clé de lecture d'une période de trouble pour l'identité espagnole en refonte. Une telle figure a permis de projeter des sens divers pour reconstruire la pensée de la guerre du point de vue de chaque clan. Antigone a été l'écran de projection pour se redéfinir en temps de crise, comme républicain, comme exilé, comme femme, comme opposant au pouvoir, ou comme 
chrétien fidèle au caudillo, chez José María Pemán. Mais certaines œuvres relèvent véritablement de la subversion du mythe : on peut citer ici l'exemple de l'œuvre d'Agustín García Calvo, dont le titre, Ismène ${ }^{11}$, augure en soi d'une vision novatrice. Cette pièce présente la discorde entre les personnages au sujet de l'installation de centrales nucléaires. L'actualisation est donc poussée à son paroxysme. En particulier, le fait que le personnage révolutionnaire de cette pièce soit plutôt Hémon qu'Antigone est très frappant. Un tel parti-pris esthétique participe d'une certaine "démythification du mythe " qui s'opère par le simple choix de mettre en avant un autre personnage qu'Antigone, et cela dès le titre. Le dramaturge a en effet décidé d'ériger Ismène en héroïne, c'est-à-dire qu'il met l'accent sur la sœur moins courageuse, qui dans la tradition, n'ose désobéir au tyran Créon. Il s'agit bien entendu d'un processus de distanciation par rapport à la tragédie de Sophocle, qui se fonde sur le choix de se centrer sur le personnage de l'autre sœur, celle qui, traditionnellement, a trop peur pour s'opposer. Ces modifications de focalisation s'accompagnent en outre d'un changement de registre pour la pièce : son auteur la définit dans le sous-titre comme une "tragicomédie ", ce qui implique un fort décalage par rapport au tragique. Le contexte d'écriture de cette œuvre est celui d'une Transition bien entamée, qui apporte son nouveau lot de préoccupations, comme l'entrée de l'Espagne dans l'OTAN en 1982, et la construction de centrales nucléaires, sujets abordés dans cette pièce. Par ailleurs, la ville de Thèbes est mentionnée, mais García Calvo procède à une actualisation sur le plan temporel, créant un fort décalage qui pourrait presque dénaturer la tragédie. En réalité, cela ne fait que révéler son pouvoir expressif et sa capacité d'adaptation, lorsque la crise politique oblige à repousser les limites de l'écriture, ou de la réécriture.

\section{De l'usage d'un double langage : quand le mythe masque un discours politiquement incorrect}

À travers un décor ancré dans l'antiquité mythique, se dévoile une épopée contemporaine, qui met à jour l'esprit d'une époque, fait de révolte, de désenchantement et de revendications. Fidèle à l'esprit de transition, un autre exemple marquant de "sub-version " se lit entre les lignes de la pièce de Riaza, qui repose sur le paradoxe suivant : Antigone est par excellence celle qui refuse la compromission chez Sophocle, or Riaza en fait la figure de la compromission. Ainsi, les thèmes d'actualité sont abordés : alors que les générations sont divisées dans le débat sur la transition démocratique, cette pièce pose la question de l'âge en rapport avec l'identité. Est-ce parce qu'elle est jeune qu'Antigone résiste et se montre aussi absolue, ou est-ce par pure conviction ? Comment se définit-on au coeur d'une époque politiquement

11 Agustín García Calvo, Ismena, Tragicomedia musical, Zamora, Lucina, 1980. 
trouble ? L'opposition radicale caractérise-t-elle uniquement ceux qui ont vécu la Guerre civile ? Ou au contraire, est-elle l'apanage de la jeunesse ? Chez Riaza, Antigone a la ferveur éphémère de la jeunesse révolutionnaire mais elle finit par "rentrer dans le rang ". L'œuvre apporte une dimension grotesque avec la figuration des cadavres de Polynice et Étéocle par des poulets plumés. La première version intitulée Las jaulas, écrite dans les années 1960, n’avait pas pu être publiée ${ }^{12}$ après avoir subi la censure en 1970, jugée trop ironique envers les tragédies grecques, et critique envers la société d’alors. Les censeurs avaient considéré que l'auteur prenait par ailleurs trop de liberté à leur goût en matière de registre de langage. La pièce, finalement éditée en 1983, reste donc très marquée par cette liberté qui explose entre les lignes. À partir de l'opposition entre Créon et sa nièce, elle reflète le conflit intergénérationnel (soit une thématique ancrée dans les débats de la décennie 1960 en Espagne) et s'inscrit dans l'esthétique du théâtre de son époque avec une démythification de la tragédie et du théâtre même. Sans apporter frontalement de critique politique, le grotesque vient caricaturer la société. Le personnage d'Antigone y est reconstruit au point d'apparaitre sous les traits d'une féministe hippy. Ce personnage contestataire va cependant finir par s'unir à Hémon et devenir une femme consensuelle et bourgeoise, déviant alors de sa trajectoire traditionnelle de jusqu'au-boutiste invétérée. À travers cette réécriture, Riaza dresse le portrait d'une génération qui selon lui s'est trahie, a renoncé à ses principes et bafoué la mémoire de ses frères morts pour leur cause. Une telle tonalité traduit la désillusion et le désenchantement ambiants quant aux espoirs nourris après quarante années de dictature. Le retour laborieux de la démocratie, concédé et orchestré par les propres membres du Régime franquiste, crée une amertume qui se traduit dans les pièces de cette période charnière. Riaza s'approprie à son tour cette histoire tragique pour dénoncer les compromissions de ceux qui étaient auparavant marginalisés par le pouvoir, tels des Antigones. La subversion trouve des échos dans le titre même, qui associe une insulte au prénom symbolisant la résistance : Antígona... jcerda! ${ }^{13}$. Ce cri injurieux envers Antigone la disqualifie totalement : l'image de la "truie " transmet l'idée d'une femme méprisable, dont les principes sont vils, sales. Ce qui caractérise le plus profondément cette figure mythique y est ridiculisé, et son identité remise en question. La traittrise d'Antigone est en effet une manière de se renier elle-même, de balayer ce qu'elle revendiquait, à savoir ses principes de jeunesse : la rébellion, la justice. Le dramaturge donne ainsi à entendre que les héritiers des vaincus de la guerre civile ont trahi les idéaux de leurs aînés lors du retour à une vie démocratique après 1975. Cette nouvelle interprétation proposée par la pièce de Riaza est novatrice en termes

12 María Josep Ragué i Arias, Lo que fue Troya, op. cit., p. 115.

13 Luis Riaza, Antígona... jcerda!, Madrid, La Avispa, 1983. 
de reconstructions identitaires. En effet, cette version déconstruit les identités et dénonce les masques de chacun. Antigone se dédouble entre une femme soumise d'un côté, et une rebelle de l'autre, comme pour figurer sur scène les deux facettes possibles de sa personne. De la même façon, Ismène, Créon et Hémon sont fondus en un seul et même personnage très ambivalent, qui porte les trois prénoms accolés. Ce parti pris scénique incite à repenser les rôles et la façon que chacun a de se définir face au dilemme. La pièce de Riaza est donc une pièce qui détruit l'identité, la substantifique moelle du mythe pour montrer qu'il n'est plus valide à ce jour, ce qui exprime clairement le degré de désenchantement ambiant.

\section{Identification et autochtonie : l'impossible retour à sa terre}

Les Antigones porteuses de revendications identitaires soulèvent des problématiques territoriales : entre territoire et terre, on lit toujours cette même blessure vis-à-vis de la terre d'origine, où chacun devrait pouvoir être enterré. La notion de territoire se trouve naturellement au cœur des revendications nationalistes qui émanent des régions espagnoles, étouffées pendant la dictature et qui ressurgissent avec fracas après 1975. Dans un tel contexte, les Antigones catalanes et galiciennes sont des figures de revendication identitaires qui émanent des marges territoriales et s'opposent au centre madrilène. Cette notion de périphérie qui s'attaque au centre du pouvoir et dénonce les dérives est soulignée par María Josep Ragué i Arias : "Depuis la Catalogne, depuis la Galice, depuis la Castille depuis l'exil, Antigone prononce un "non " contre la guerre civile, contre la dictature ${ }^{14}$. " En effet, dans les dernières années du franquisme, on assiste à une renaissance littéraire des langues régionales, notamment en catalan, en basque et galicien, langues qui vont aussi s'approprier l'histoire d'Antigone. Cela donne naissance à des Antigones dites "nationales" (au sens des nationalismes régionaux) qui sont des pièces au contenu idéologiquement très marqué. Elles intègrent des éléments contemporains, font la part belle aux cultures régionales et aux luttes actuelles, comme les révoltes étudiantes de la fin des années 1960. L'œuvre de Maria José Queizán, intitulée Antigone, la force du sang $g^{15}$, constitue une version très novatrice de la tragédie sophocléenne, et s'inscrit en rupture vis-à-vis des précédentes réécritures ibériques. En premier lieu, cette œuvre rédigée en galicien s'inscrit dans le contexte du développement de l'État des autonomies, alors que se consolide la démocratie qui permet un renouveau culturel dans les années 1980. Celui-ci s'accompagne de fortes revendications autonomistes au sein de la communauté galicienne, dont la dramaturge est

14 María Josep Ragué i Arias, «Del mito contra la dictadura al mito que denuncia la violencia y la guerra. " Foro hispánico : revista hispánica de Flandes y Holanda, no 27, 2005.

15 María Xosé Queizán, Antígona, a forza do sangue, Vigo, Xerais, 1989. 
originaire. Dans sa pièce, le pouvoir illégitime et oppresseur est celui des Espagnols - représentés par Créon - sur la Galice (qui correspond au territoire de Thèbes). Il s'agit d'une recréation très libre du mythe, dont l'objectif principal, selon les dires de l'écrivaine, est de " transformer l'héritage antique en héroïne moderne ${ }^{16} »$. Elle revendique alors une Antigone autonomiste et féministe ${ }^{17}$, qui transcende le caractère individuel de l'éthique du personnage pour " aborder le social » et "devenir le symbole d'une culture et d'une nation ». Par conséquent, l'appropriation très originale de la figure tragique induit une réécriture elle-même marginale au sein des Antigones espagnoles. Ce décalage avec les autres est assumé par Queizán, qui reprend à son compte un mythe à dimension universalisante pour sa revendication identitaire. À première vue, cela peut paraitre paradoxal, mais cette Antigone-là relève du besoin d'appropriation du mythe dans la langue galicienne, et l'écriture parvient à faire fusionner universalisme et marginalité. Dans son introduction à l'œuvre, Queizán déclare d'ailleurs qu'écrire cette œuvre dans la langue galicienne était un devoir. En définitive, les réceptions catalane et galicienne du mythe d'Antigone incitent à s'interroger sur la question de son interculturalité. La réécriture dans une langue régionale suscite des réflexions plus complexes encore, étant donné le contexte historico-politique : cela peut supposer en particulier une revendication à caractère communautariste. La répression des langues régionales pendant la dictature avait conditionné la réception des ouvres, et induit un fort décalage temporel entre le contexte d'écriture et le temps de la diffusion. Ce fut le cas en particulier pour la pièce de Salvador Espriu, comme évoqué précédemment, qui avait été censurée dans un premier temps du fait de la répression de la langue catalane. La censure d'une langue a une forte portée symbolique, en ce qu'elle suppose de nier la parole de l'opposant dans sa forme même. Bâillonner celui qui s'oppose, c'est précisément le vain essai de Créon, qui tente de raisonner Antigone, lui promettant de se taire et de faire taire les gardes si elle se plie aux ordres de son oncle et renonce à enterrer son frère. Historiquement, le rôle particulier de la communauté catalane dans la Guerre civile a entraîné ce silence imposé par la censure du véhicule linguistique. Ces auteurs ont considéré que la meilleure réponse serait sans doute d'écrire une Antigone dans une langue communautaire... Enfin, au-delà de la langue elle-même, le fond du message transmis par certaines Antigones " des marges ", rejoint l'image d'Antigone comme figure de l'opprimée. Cette symbolique est à mettre en lien avec les problématiques identitaires faisant débat à cette époque très agitée sur le plan politique. Par conséquent, il est essentiel de montrer la portée idéologique qu'entraîne la transposition du mythe d'Antigone dans

16 Ibid.

17 Bernard Sicot, "Les Antigones contemporaines (de 1945 à nos jours) ", Bulletin hispanique, 2011, p. 816-821. 
une autre langue que le castillan pendant la dictature et même dans les années qui suivent. Les messages politiques s'écrivent donc entre les lignes du mythe d'Antigone.

\section{Conclusion}

En définitive, une telle résurgence de la figure d'Antigone dans le théâtre espagnol à partir de l'après-guerre s'explique par son lien intrinsèque avec le genre tragique, repris et déconstruit en Espagne durant cette période troublée, mais aussi parce qu'elle symbolise l'opposition à la loi tyrannique et la rébellion, ainsi que la fidélité à une loi supérieure (liée à la religion, ou l'amour filial). Cela indique que cette période de crise historique a suscité des interrogations identitaires, auxquelles les écrivains ont répondu via ce mythe. Antigone est un personnage de l'absolu, de la pureté, qui refuse le compromis. Mais durant les balbutiements de la démocratie retrouvée, elle prend plusieurs visages et incarne tantôt la réconciliation, tantôt le désenchantement quant à la possibilité d'une unité nationale fondée sur des ciments identitaires. Le personnage, plongé dans un décor contemporain, prêche la réunion de camps opposés, le pansement des blessures identitaires, et offre une main tendue vouée à reconstruire l'idée de nation, présentée comme une famille à travers cette tragédie, tout en n'oubliant pas le passé. Antigone se définit de manière absolue car elle est face à sa finitude, face à sa mort certaine, une mort à laquelle elle s'est elle-même condamnée par son acte, et qu'elle actualisera par son suicide. Dans ces réécritures espagnoles, elle regarde alors son monde pour la dernière fois, sans concessions. La littérature dramatique s'est constituée en espace propice à une réflexion sur l'identité déchirée, remise en cause par la lutte fratricide. Ce recours massif à l'histoire d'Antigone n'est pas exempt de déconstructions : la plupart des auteurs ont été au-delà de la victimisation et ont mis en scène l'inanité du conflit pour prôner une possible réconciliation de la nation - passant par une " juste mémoire »-, comme c'est le cas pour Bergamín et Espriu. Récrire Antigone au XXe siècle, sous la dictature franquiste, c'est réfléchir au pouvoir exercé sans limites : c'est d'ailleurs le point commun entre Antigone et Créon, à savoir le caractère absolu et jusqu'au-boutiste de leur action. Or le délicat passage de la dictature à la démocratie suppose la convergence de forces opposées, qui ne peut avoir lieu qu'à la condition du compromis. Les frustrations que crée ce compromis entre 1975 et 1978, critiqué pour son "pacte de silence ", sont à l'origine d'une nouvelle résurgence de la tragédie antigonienne dans les productions dramaturgiques espagnoles. Les reprises du mythe dialoguent et s'opposent, se répondent, participant à la projection sur scène d'un débat éminemment contemporain.

Les paradigmes de la guerre de Troie et des sept contre Thèbes, exploités dans la période de post-guerre civile, et à chaque fois que ce conflit irradie l'actualité, continuent d'inspirer les œuvres littéraires qui s'emparent de ques- 
tionnements identitaires. C'est le cas en effet au sujet de conflits plus récents, comme la guerre du Kosovo, les combats en Tchétchénie, au Liban ou en Irak. Ce qui montre l'aptitude toute particulière des figures mythiques à représenter les refontes identitaires en période de trouble.

\author{
Fanny Blin \\ EA 3656 AMERIBER \\ Université Bordeaux Montaigne \\ fblin@u-bordeaux3.fr
}

\begin{abstract}
Résumé
Au lendemain de la Guerre Civile espagnole, alors que le régime franquiste tendait à imposer un discours officiel autour de l'" identité nationale ", les productions dramaturgiques ont reflété le trouble historique. Celui-ci avait ébranlé les cadres de définition identitaires, à échelle individuelle et collective. Grâce au principe d'identification du spectateur, le théâtre favorisait l'expression de cette crise. Notamment, la figure d'Antigone a servi d'écran de projection en cette période de trouble, à travers de nombreuses réécritures de l'œuvre de Sophocle, suscitées par un contexte qui faisait écho à l'histoire fratricide de Thèbes. Sa tragédie est celle d'un dilemme, qui la pousse à se définir, à " choisir son camp ", à l'instar des Espagnols à cette période. La mise en scène du conflit intérieur - qui s'empare à la fois de l'unité du sujet et de l'unité nationale - structure alors les questionnements identitaires, autour de trois axes principaux : la lutte fratricide, l'exil, et les régionalismes. La définition de soi ne prenant sens que dans la confrontation à l'Autre, cette figure de la résistance inspire les dramaturges, qui ré-interprètent la tragédie à l'aune des troubles du XXe siècle. La comparaison de ces réécritures contemporaines du mythe en Espagne révèle que ce personnage a servi de porte-voix à une époque de censure implacable, pour des (re)constructions identitaires qui passent par la déconstruction du mythe.
\end{abstract}

\title{
Mots-clés
}

Antigone, guerre civile, identification, dictature, nationalisme.

\begin{abstract}
After the Spanish Civil War, while Franco was promoting a unitary and nationalist idea of "Spanish identity", playwrights recreated the historical strife through their works. As the frameworks for a definition of identity had been shattered, theatre enabled the expression of this crisis, thanks to the identification process that is inherent in tragedy. The figure of Antigone particularly haunted the Spanish stage, through more than twenty contemporary versions of Sophocles' play during the Franco era. Indeed, Antigone's claim fitted perfectly with the expression of the ideological division of the time. Within such a troubled history, these plays emerge as a way to speak again after power had imposed silence, with this Greek character who advocates for unity among a divided family. Most of the "Spanish Antigones" delve into three main topics: exile, the fratricidal struggle, and regionalisms. Comparing these contemporary rewritings of the myth in Spain reveals this character as the spokesperson of those who perceived themselves as " the losers " of the Civil War, allowing for a construction of new identities through a deconstruction process of the myth.
\end{abstract}

\section{Keywords}

Antigone, civil war, identification, dictatorship, nationalism. 